\title{
Network Analysis of Chaotic Dynamics in Fixed-precision Digital Domain
}

\author{
Chengqing Li \\ College of Computer Science and \\ Electronic Engineering, Hunan University, \\ Changsha 410082, Hunan, China \\ chengqingg@gmail.com
}

\author{
Jinhu Lu \\ School of Automation Science \\ and Electrical Engineering, \\ Beihang University, \\ Beijing 100083, China
}

\author{
Guanrong Chen \\ Department of Electronic Engineering, \\ City University of Hong Kong, \\ Hong Kong SAR, China
}

\begin{abstract}
When implemented in the digital domain with time, space and value discretized in the binary form, many good dynamical properties of chaotic systems in continuous domain may be degraded or even diminish. To measure the dynamic complexity of a digital chaotic system, the dynamics can be transformed to the form of a state-mapping network. Then, the parameters of the network are verified by some typical dynamical metrics of the original chaotic system in infinite precision, such as Lyapunov exponent and entropy. This article reviews some representative works on the network-based analysis of digital chaotic dynamics and presents a general framework for such analysis, unveiling some intrinsic relationships between digital chaos and complex networks. As an example for discussion, the dynamics of a state-mapping network of the Logistic map in a fixed-precision computer is analyzed and discussed.
\end{abstract}

Index Terms-arithmetic domain, chaotic cryptography, chaotic dynamics, Logistic map, state-mapping network.

\section{INTRODUCTION}

$\mathbf{C}$ Haos is typically defined in the continuous domain, for which many interesting dynamical properties hold only in infinite precision. Yet, implementing a chaotic map in a finite-precision device is an inevitable process for any of its real applications or for any simulation in the digital world. Therefore, the dynamical properties of a digitized chaotic map have been the main concern in nonlinear science and chaotic cryptography. When iterating a chaotic map in the digital domain, its orbit is perturbed by the quantization errors. Consequently, not a real-valued chaotic orbit but only a discretized pseudo-orbit is obtained. Such pseudo-orbits are considered useful due to the well-known shadowing lemma, which says that every pseudo-orbit obtained via computer simulation has a true error-free orbit nearby. The shadowing lemma justifies the use of computers to study chaotic systems and their dynamics. As a consequence, many efforts were devoted to designing pseudo-random number generators utilizing chaotic maps [1]. Nevertheless, it was argued [2], [3] that the dynamics of digital chaotic maps are degraded to various extents and such dynamical degradation can create big problems to some applications, for example thwarting the security of the chaos-based cryptosystems as iterating a

This work was supported by the National Natural Science Foundation of China (nos. 61772447, 61532020). degraded chaotic map generates a short-period pseudo-random number sequence with a non-negligible probability [4]-[6].

Despite the theoretic elegancy of the shadowing lemma, it becomes rather misleading when one looks at the system as a whole: all shadowing orbits expressed in a particular finite precision constitute only a negligible part of the whole set of true orbits. This is not surprising because the states that a pseudo-orbit can visit are countable, but in continuous domain they are not. Even worse is that, in a finite-state space, all pseudo-orbits will eventually become periodic and the period is bounded by the number of the states therein. Thus, to what extent the finite-precision orbits can still retain the dynamical properties of the original chaotic system calls for quantitative analysis of the set of pseudo-orbits. This turns out to be a rather complicated problem and cannot be easily handled by existing theories and techniques. In fact, most existing work focus on the average behaviors of all pseudo-orbits, but still cannot tell anything more about the pseudo-orbits within different fine structures. In [7], some fine structures in such pseudo-orbits implemented with fixed-point arithmetic are revealed and discussed.

Recently, complex networks as an emerging research field can provide useful tools for studying the dynamics of chaotic time series, first attempted in [8]. Essentially, some earlier work studying the dynamics of digital chaos can be attributed to the notion of complex networks [9]. Specifically, in 1986 Binder and Jensen built a state-mapping network (SMN) of the Logistic map and reported that the counterparts of some metrics on measuring the dynamics of continuous chaos, such as Lyapunov exponent, remain to work as usual [10]. In the following years, Binder reported some more work and findings: the relationship between a limit cycle of the Logistic map and its control parameter [11]. Binder and Okamoto studied the shadowing of unstable periodic orbits (UPO) with the corresponding limit cycles [12]. In [13], Hsu investigated the global properties of nonlinear dynamical systems by cellto-cell mapping. Kurths and his team presented an analytical framework for recurrence network analysis of time series [14], and then further studied the geometry of chaotic dynamics from the perspective of complex networks [15]. In [16], Frahm and Shepelyansky analyzed the small-world properties of Ulam's networks of the Chirikov standard map and the 
Arnold cat map, showing that the number of degrees of separation grows logarithmically with the network size. In [17], Shreim et al. studied the dynamics of series generated by cellular automata, using the parameters of the associate state-mapping network. The properties of Chebyshev map on a finite field was investigated with a directed network (graph) by Gassert [18]. Recently, the functional graph associated with the iterations of the Chebyshev polynomials over a finite field was quantitatively analyzed by Qureshi and Panario [19]. It should be noted, however, that networks typically exhibit selforganizing fractals (and multifractals) and chaos by themselves when they are sufficiently large and complex [20].

This paper reviews the existing complex-network-based dynamic analysis of digital chaotic systems, using the Logistic map as a typical example. A general framework for analysis is presented. Furthermore, it discusses how the structure of the SMN of the Logistic map is changed with the fixed-point arithmetic precision on a computer. Some fractal phenomena and power-law in-degree distributions are observed when the precision is incrementally increased.

The rest of the paper is organized as follows. Section II briefly reviews the existing dynamic analysis of digital chaotic systems from the general viewpoint of complex networks. Some special properties of the SMN of the Logistic map are reported in Sec. III. The last section concludes the paper.

\section{BRIEF SURVEY ON COMPLEX-NETWORK-BASED DYNAMIC ANALYSIS OF DIGITAL CHAOTIC MAPS}

Following Ulam's method proposed in [21, p. 74], the statemapping network of the Logistic map

$$
f(x)=\mu \cdot x \cdot(1-x)
$$

was built by the following three steps in [22], [23]: 1) divide the phase interval into some subintervals; 2) each subinterval is considered as a node; 3) two nodes are linked by a directed edge if a (predefined) map relationship between the corresponding intervals exist.

Once the network is built, various parameters can be calculated to reveal some rules similar to that obtained in the infinite resolution domain. The general framework of analyzing the dynamical properties of chaotic systems can be shown by the four stages in Fig. 1.

Selecting nodes $\rightarrow \begin{aligned} & \text { Establishing link } \\ & \text { between nodes }\end{aligned} \rightarrow \begin{aligned} & \text { Defining network } \\ & \text { parameters }\end{aligned} \rightarrow \begin{aligned} & \text { Comparing with other } \\ & \text { recognized metrics }\end{aligned}$

Fig. 1. Framework of network-based analysis of dynamical systems.

In [23], the phase space of a chaotic map $g(x)$ is equally divided into $N$ disjoint sub-space, and each sub-space is defined as a node. Given an initial state, $x_{0}$, the $i$ th node is directly linked to the $j$ th node if and only if $x_{0}$ and $g\left(x_{0}\right)$ belong to the two corresponding sub-spaces, respectively. Iterating the chaotic system from the current state $T$ several times and linking the related nodes in a similar manner, the space network is established.
Figure 2 depicts the largest in-degree of the corresponding space network under different control parameters with five network sizes. From Fig. 2, one can see that the largest indegree obviously follows a power-law distribution with the same scaling exponent for $N \in\{2000,10000\}$. In contract, there are two scaling exponents for $N \in\{1000,5000\}$. This may be caused by the non-uniform distributions of the floatingpoint numbers in different sub-spaces.

Figure 3 presents the average path length of the space network of the Logistic map under different control parameters with five network sizes. Observing Fig. 3, one can see that the changing trend of the average path length with respect to the control parameter matches that of the Lyapunov exponents of the Logistic map to a relatively high extent, demonstrating that the parameters of the phase network can serve as an alternative measure for detecting the 'edge of chaos' in dynamical systems.

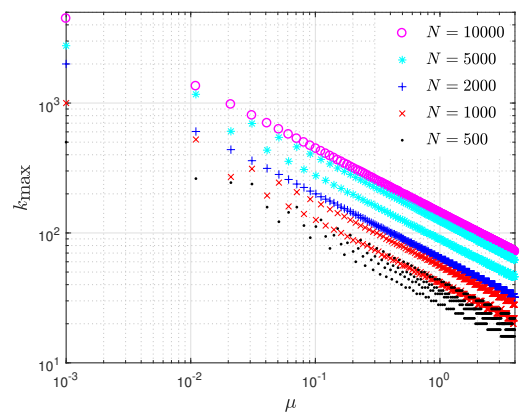

Fig. 2. The largest in-degree $k \max$ of the space network of the Logistic map in five network sizes.

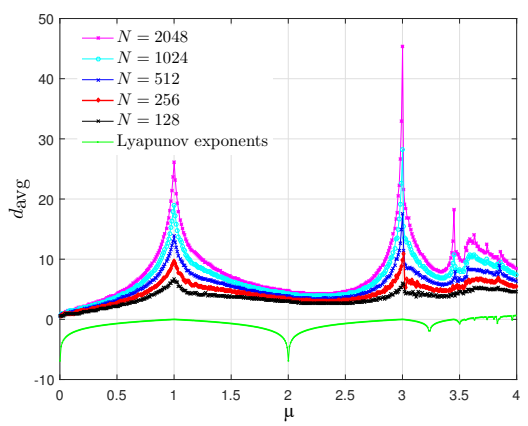

Fig. 3. The average path length of the space network of the Logistic map with respect to the control parameter $\mu$ for five network sizes.

In [24], the time series generated by a chaotic system is transformed to a network (graph) using the mechanism of horizontal visibility graph, where every state is considered as a node and two arbitrary data points are linked if and only if they satisfy the geometric 'visibility' criterion. Then, a parameter called "network entropy" is defined. As shown in Fig. 4, the changing trend of the network entropy of the associate network of the Logistic map can mimic that of the Lyapunov exponent. In [25], two arbitrary states of a time series are connected as a pair of nodes if the linear interpolation between them is larger than the values of all intermediate points. The distribution of 


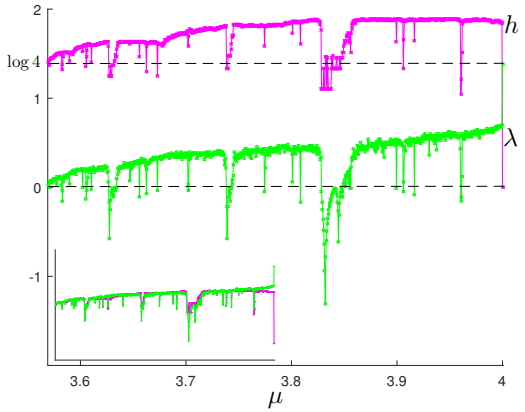

Fig. 4. Dynamical demonstration of the Logistic map under two metrics: horizontal visibility network entropy $h$ and Lyapunov exponent $\lambda$.

different subgraphs is shown to be a sensitive measure of the underlying dynamics. As shown in Fig. 5, motif distribution can be used to distinguish broad classes of chaotic dynamical systems, to which the Logistic map belongs.

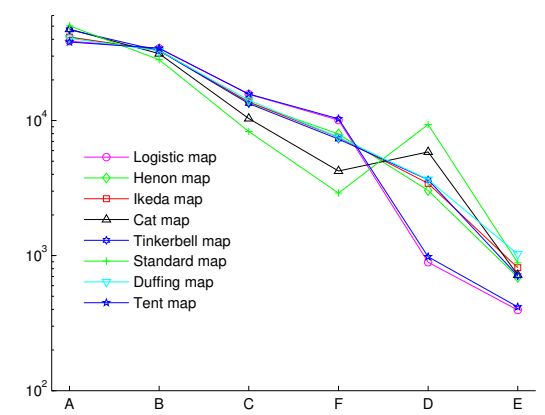

Fig. 5. Subgraph ranks of different types of time series, where $A, B, C, D$, $E$, and $F$ represent 6 different possible undirected subgraphs of size 4 .

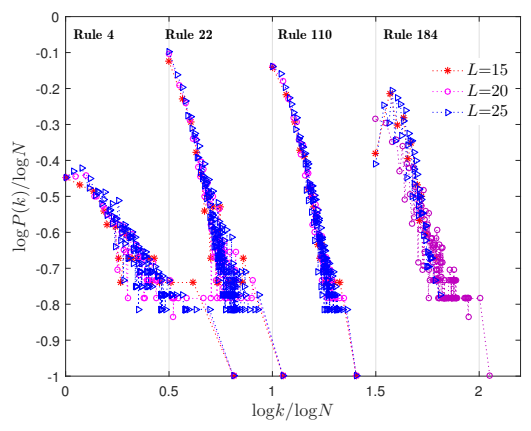

Fig. 6. In-degree distribution $P(k)$ for four rules of CA with three values of CA having size $L$, where $N=2^{L}$.

In [17], the state-space network of the sequential dynamical systems is analyzed, which is composed by 1-D cellular automata (CA) with rules involving two colors and the nearest neighbors, where every binary state of size $L$ is viewed as a node of a directed network and the evolution relation between two nodes builds a link connecting them. Based on analytic analyses and experimental results on some selected rules, it was claimed that the co-appearance of nontrivial scalings in both the hub sizes and the path diversity of the state-phase networks can separate simple dynamics from the more complex ones found in CAs falling into Wolfram's classes III and IV (see the in-degree distribution for four rules shown in Fig. 6). Yet, the validity of such classification method was questioned in [26], which plots the distribution of the two parameters corresponding to all elementary rules of 1-D CA falling into the same Wolfram's class. How to accurately measure the dynamical complexity of a chaotic system with the methodology of complex networks needs further investigation.

It has been observed that, in the field of nonlinear science, many did not care about how their computers perform fixedpoint or floating-point arithmetic operations, but implemented a chaotic system in the computer just like a black box. Most related research on dynamical analysis based on network (graph) adopt the Logistic map as test objects [1], [2], [10], [14], [15], [22]-[25], where the real structure of SMN of the Logistic map in the computer has been unfortunately ignored.

\section{DYNAMICAL ANALYSIS OF A STATE-MAPPING NETWORK OF THE LOGISTIC MAP ON COMPUTER}

Now, consider the following state-mapping network of the Logistic map: every value in the $n$-bit arithmetic computing precision is identified as a node; a directed edge between nodes $u$ and $v$ exists if and only if the former node is mapped to the latter one by the Logistic map. The following key parameters of the space network were studied, revealing the relationships among the Logistic map (1), the computing precision and the control parameter.

- Loop: (also called a self-loop) is an edge that connects a node to itself.

- A directed cycle in a directed graph is a sequence of nodes, starting and ending at the same node such that, for each pair of two consecutive nodes in the cycle, there exists an edge directed from the previous node to the later one.

- A weakly connected component (WCC) is a maximal subgraph of a directed graph such that there is a path between every pair of nodes $u$ and $v$ in the subgraph, regardless of the direction of edges.

To observe the properties of SMN of the Logistic map, the software platform Cytoscape is used to draw some space networks under some given parameter values. The space networks of the Logistic map of $\mu=\frac{121}{2^{5}}$ under 5-bit and 6-bit fixed-point arithmetic are shown in Fig. 7a) and b), respectively, where a round quantization is adopted. To show the rule of the network with respect to the increase of the precision more clearly, the state network of the Logistic map under 6-bit precision with the same value of the control parameter is shown in Fig. 8. When the precision is further increased to 12, the network becomes the case shown in Fig. 9, demonstrating some fractal phenomena, as studied in [27]. As shown in Fig. 10, the in-degree distribution of $F_{n}$ approaches a power-law more steadily as $n$ increases, where $F_{n}$ denotes the function of Logistic map (1) implemented under $n$-bit precision. 
TABLE I

INTERMEDIATE VALUES OF THE CALCULATING LOGISTIC MAP IN BINARY16.

\begin{tabular}{c|c|c|c|c}
\hline$x$ & $1-x$ & $1-(1-x)$ & $f(x)$ & $f(1-x)$ \\
\hline 0.0099945068359375 & 0.98974609375 & 0.01025390625 & 0.037384033203125 & 0.038360595703125 \\
\hline 0.04998779296875 & 0.94970703125 & 0.05029296875 & 0.179443359375 & 0.1805419921875 \\
\hline 0.0899658203125 & 0.90966796875 & 0.09033203125 & 0.309326171875 & 0.310546875 \\
\hline 0.0999755859375 & 0.89990234375 & 0.10009765625 & 0.340087890625 & 0.340576171875 \\
\hline 0.199951171875 & 0.7998046875 & 0.2001953125 & 0.6044921875 & 0.60498046875 \\
\hline 0.289794921875 & 0.7099609375 & 0.2900390625 & 0.77783203125 & 0.7783203125 \\
\hline 0.389892578125 & 0.60986328125 & 0.39013671875 & 0.89892578125 & 0.8994140625 \\
\hline 0.489990234375 & 0.509765625 & 0.490234375 & 0.9443359375 & 0.94482421875 \\
\hline
\end{tabular}

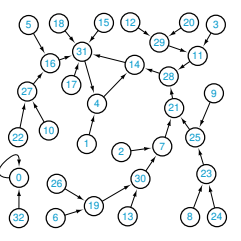

a)

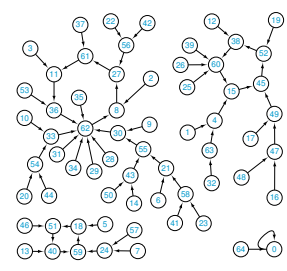

b)
Fig. 7. The state network of the Logistic map with $\mu=\frac{121}{2^{5}}$ under round quantization: a) 5-bit precision; b) 6-bit precision.

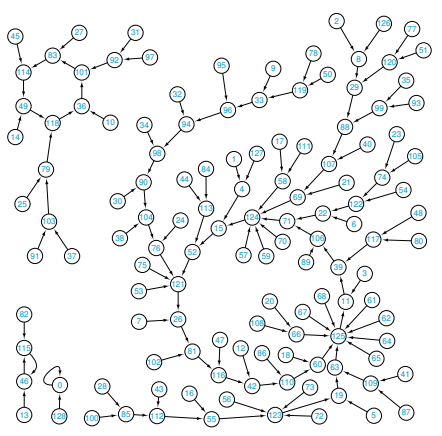

Fig. 8. The state network of the Logistic map under 6-bit precision and round quantization, where $\mu=\frac{484}{2^{7}}$.

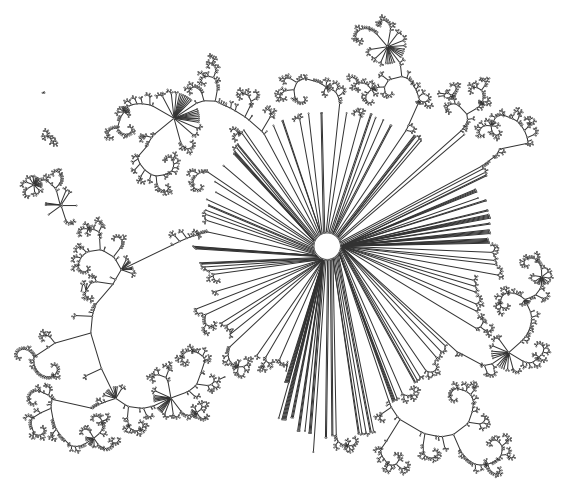

Fig. 9. The state network of the Logistic map under 12-bit precision and round quantization, where $\mu=\frac{15488}{2^{12}}$.

Based on the above-verified results, one could infer more on the properties of the space network of the Logistic map, as listed below.

- Each space network is composed of a small number of

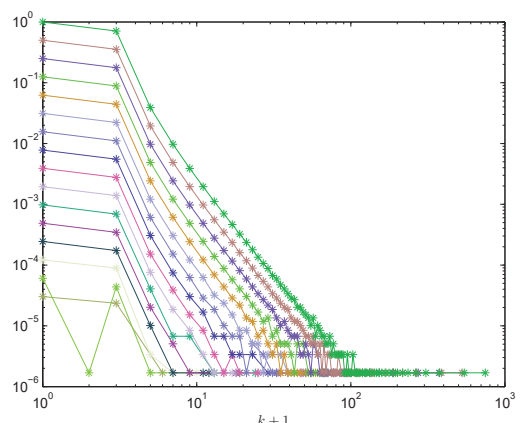

Fig. 10. In-degree distribution of the sate networks of $F_{5} \sim F_{20}$.

weakly connected components;

- Each weakly connected component has one and only one self-loop or cycle;

- In each cycle of length longer than 2, there is at least one node with a large in-degree;

- Let $\left(D_{n} / 2^{n}\right)$ denote the node with the largest degree in the state network of $F_{n}(x)$. Then,

$$
D_{n+1} \in\left\{2 \cdot D_{n}, 2 \cdot D_{n}+1,2 \cdot D_{n}-1\right\},
$$

where $n \geq n_{\mu}, \mu=\frac{N_{\mu}}{2^{n_{\mu}}}, N_{\mu}, n_{\mu} \in \mathbb{Z}^{+}$.

- One weakly-connected component dominates the whole network, i.e., the size of the component accounts for more than half of the size of the whole network. Among all the connected components, there is a clearly decreasing order.

In the floating-point arithmetic domain, the symmetric property of the Logistic map does not hold in general [3]. To demonstrate this point, some intermediate data obtained from calculating the Logistic map using half-precision floating-point format (binary16) are shown in Table I.

\section{CONCLUSION}

This paper has reviewed some representative methods for measuring the dynamical complexity of digitized chaotic systems from the perspective of complex networks. The general framework of such analyses is established to facilitate the comparison of performances of comparable analytic methods. Some subtle properties of the state-mapping network of the Logistic map operating on a fixed-point arithmetic computer have been analyzed and discussed. This paper demonstrates that the methodology of complex networks provides a novel tool for studying the complex dynamics of digital chaos. 


\section{REFERENCES}

[1] S. C. Phatak and S. S. Rao, "Logistic map: A possible random-number generator," Physical Review E, vol. 51, no. 4, pp. 3670-3678, 1995.

[2] K. Persohn and R. Povinelli, "Analyzing logistic map pseudorandom number generators for periodicity induced by finite precision floatingpoint representation," Chaos Solitons \& Fractals, vol. 45, no. 3, pp. 238-245, 2012.

[3] C. Li, B. Feng, S. Li, J. Kurths, and G. Chen, "Dynamic analysis of digital chaotic maps via state-mapping networks," IEEE Transactions on Circuits and Systems I: Regular Papers, vol. 66, no. 6, 2019.

[4] C. Li, D. Lin, J. Lü, and F. Hao, "Cryptanalyzing an image encryption algorithm based on autoblocking and electrocardiography," IEEE MultiMedia, vol. 25, no. 4, pp. 46-56, 2018.

[5] C. Li, D. Lin, B. Feng, J. Lü, and F. Hao, "Cryptanalysis of a chaotic image encryption algorithm based on information entropy," IEEE Access, vol. 6, pp. 75 834-75 842, 2018.

[6] D. Yoshioka, "Properties of chebyshev polynomials modulo $p^{k}$," IEEE Transactions on Circuits and Systems II: Express Briefs, vol. 65, no. 3, pp. 386-390, 2018.

[7] S. Li, G. Chen, and X. Mou, "On the dynamical degradation of digital piecewise linear chaotic maps," International Journal of Bifurcation and Chaos, vol. 15, no. 10, pp. 3119-3151, 2005.

[8] J. Zhang and M. Small, "Complex network from pseudoperiodic time series: topology versus dynamics," Physical Review Letters, vol. 96, no. 23, p. 238701, 2006.

[9] Y. Zou, R. V. Donner, N. Marwan, J. F. Donges, and J. Kurths, "Complex network approaches to nonlinear time series analysis,' Physics Reports, vol. 787, pp. 1-97, 2019.

[10] P. M. Binder and R. V. Jensen, "Simulating chaotic behavior with finitestate machines," Physical Review A, vol. 34, no. 5, pp. 4460-4463, 1986.

[11] P. Binder, "Limit-cycles in a quadratic discrete iteration," Physica D, vol. 57, no. 1-2, pp. 31-38, 1992.

[12] P.-M. Binder and N. H. Okamoto, "Unstable periodic orbits and discretization cycles," Physical Review E, vol. 68, no. 4, p. 046206, 2003.

[13] C. S. Hsu, "Global analysis by cell mapping," International Journal of Bifurcation and Chaos, vol. 2, no. 4, pp. 727-771, 1992.

[14] J. F. Donges, J. Heitzig, R. V. Donner, and J. Kurths, "Analytical framework for recurrence network analysis of time series," Physical Review E, vol. 85, no. 4, p. 046105, 2012.

[15] R. V. Donner, J. Heitzig, J. F. Donges, Y. Zou, N. Marwan, and J. Kurths, "The geometry of chaotic dynamics - a complex network perspective," European Physical Journal B, vol. 84, no. 4, pp. 653-672, 2011.

[16] K. M. Frahm and D. L. Shepelyansky, "Small world of Ulam networks for chaotic Hamiltonian dynamics," Physical Review E, vol. 98, no. 3, p. art. no. 032205, 2018.

[17] A. Shreim, P. Grassberger, W. Nadler, B. Samuelsson, J. E. S. Socolar, and M. Paczuski, "Network analysis of the state space of discrete dynamical systems," Physical Review Letters, vol. 98, no. 19, p. 198701, 2007.

[18] T. A. Gassert, "Chebyshev action on finite fields," Discrete Mathematics, vol. 315, pp. 83-94, 2014.

[19] C. Qureshi and D. Panario, "The graph structure of chebyshev polynomials over finite fields and applications," Designs, Codes and Cryptography, 2019.

[20] C. Song, S. Havlin, and H. A. Makse, "Self-similarity of complex networks," Nature, vol. 433, no. 7024, pp. 392-395, 2005.

[21] S. M. Ulam, Problems in Modern Mathematics, ser. Interscience tracts in pure and applied mathematics. New York, USA: John Wiley \& Sons, 1964.

[22] T. Iba, "Hidden order in chaos: The network-analysis approach to dynamical systems," in Proceedings of the Eighth International Conference on Complex Systems, 2011, pp. 769-783, http://necsi.edu/events/iccs2011/papers/70.pdf.

[23] F. Kyriakopoulos and S. Thurner, "Directed network representation of discrete dynamical maps," in International Conference on Computational Science, ser. Lecture Notes in Computer Science, vol. 4488. Springer, 2007, pp. 625-632.

[24] B. Luque, L. Lacasa, F. J. Ballesteros, and A. Robledo, "Feigenbaum graphs: a complex network perspective of chaos," PLoS One, vol. 6, no. 9, p. art. no. e22411, 2011.

[25] X. Xu, J. Zhang, and M. Small, "Superfamily phenomena and motifs of networks induced from time series," Proceedings of the National Academy of Sciences, vol. 105, no. 50, pp. 19601-19605, 2008.
[26] C. Xu, C. Li, J. Lü, and S. Shu, "On the network analysis of the state space of discrete dynamical systems," International Journal of Bifurcation and Chaos, vol. 27, no. 4, p. art. no. 1750062, 2017.

[27] J.-L. Liu, Z.-G. Yu, and V. Anh, "Topological properties and fractal analysis of a recurrence network constructed from fractional brownian motions," Physical Review E, vol. 89, no. 3, p. 032814, 2014. 